\section{$\S 1$. Burst X-Ray Detection Using RMSAFE}

Yamanishi, H., Miyake, H., Matsuoka, K., Isobe, M.

The function of the radiation monitoring system RMSAFE (Radiation Monitoring System Applicable to Fusion Experiments) is well verified to detect burst radiation, that is, radiation generated suddenly and explosively. When an increase in $50 \mathrm{~ms}$ integrated count from a radiation monitor, which is recorded and updated every $10 \mathrm{~ms}$ in the system CPU, is encountered to exceed a pre-set level, RMSAFE recognizes it as an outbreak of burst radiation and alters its recording mode so that the burst event data is saved in a specified file. In this study, we detected X-rays arising from Compact Helical System (CHS), a high-temperature plasma experimental device, in order to verify that RMSAFE is able to detect radiation bursts successfully and accurately.

Because the CHS does not make use of a superconducting coil, the magnetic field rises and falls during each plasma experiment. The typical pattern of the magnetic field during a plasma shot is an increase for 0.8 $\mathrm{s}$, followed by a steady level for 0.6-1.2 seconds, and finally a decrease for $0.8 \mathrm{~s}$. The electrons in the plasma vacuum vessel are accelerated by this varying magnetic field. When the hydrogen gas in the vacuum vessel is of low density, the accelerated electrons become run-away electrons free from collision, which then do finally hit somewhere in the vacuum vessel, producing bremsstrahlung X-rays. These X-rays were used in the present study to test the burst detection capabilities of RMSAFE.

When a burst X-ray event was detected in the torus hall, increases in the count were sometimes observed even at posts WN, WB, IA, WC, IC, WF. Increases in the dose of radiation due to $\mathrm{X}$-rays from $\mathrm{CHS}$ were observed concurrently at various observation points in several plasma shots (see Fig. 1). Approximately 90 events were observed in the fiscal year 2000. Doses greater than 0.001 $\mathrm{mSv}$ were observed in the torus hall in 35 events. The weekly accumulated values of radiation dose observed by
RMSAFE in the CHS torus hall were consistent with the results of integrated dose measurements by thermoluminescent dosimeter (TLD) and by radiophotoluminescent dosimeter (RPLD), and furthermore, the general decreasing tendency of the observed dose with the distance from the CHS torus was clearly seen, though detailed radiation patterns might be dependent on source plasma and other conditions. These results support our conclusion that RMSAFE is able to successfully detect burst X-rays.

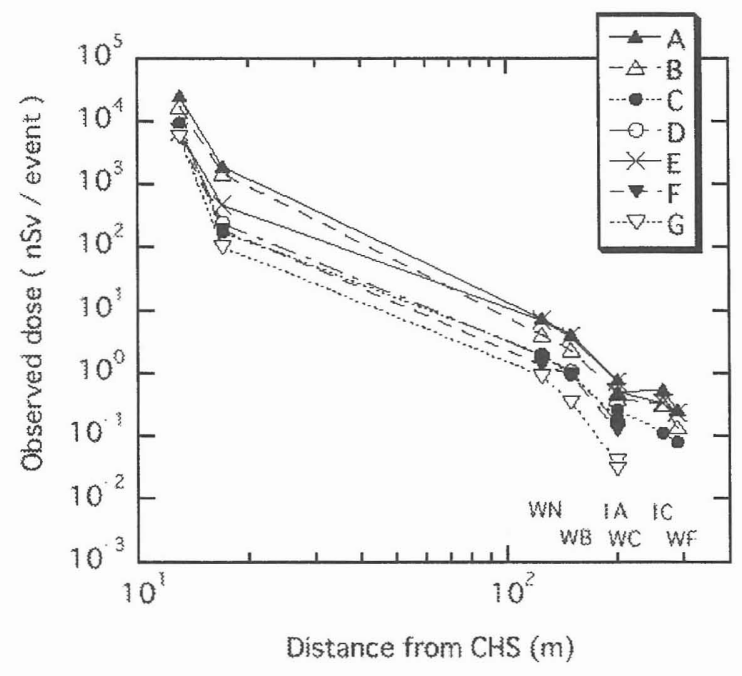

Fig. 1 Relationship between observed dose and distance from CHS 7 events $(A \sim G)$, which were detected the dose greater than $0.005 \mathrm{mSv}$ in the CHS torus hall.

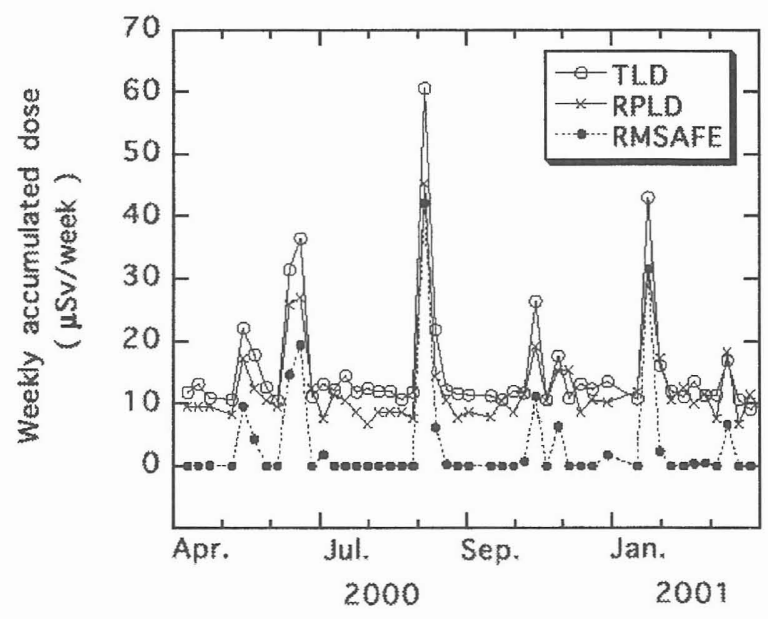

Fig. 2 Comparison of weekly measured dose between RMSAFE and two integrating dosimeters. 